\title{
An attempt to demonstrate magnetic compass orientation in two species of mammals
}

\author{
ROBERT C. MADDEN \\ Marymount College, Tarrytown, New York \\ and \\ JOHN B. PHILLIPS \\ Yale University, New Haven, Connecticut
}

\begin{abstract}
An attempt was made to train two species of small mammals (the bush opossum, Monodelphis domesticus, and the Djungarian hamster, Phodopus sungorus) to search for food in the direction indicated by an earth-strength magnetic field. Five opossums and 4 hamsters were tested in a cross-shaped arena whose four arms faced geographic north, south, east, and west. A set of coils generated a magnetic field whose north pole could be randomly pointed toward any arm. A subject was given a food reward if it entered the arm indicated by the north direction of the field. In 12 series of 73 to 1,127 trials each, the subjects were unable to select the appropriate arm with a frequency greater than random. This result contrasts strongly with a variety of published studies on mammals, birds, and other vertebrates that have indicated good ability to determine direction from the earth's magnetic field.
\end{abstract}

Most reported responses of animals to the earth's magnetic field fall into one of two categories: (1) magnetic compass responses-orientation to the direction of the earth's field; or (2) gamma-level responses-changes in orientation or activity correlated with minute fluctuations or disturbances in the earth's field. This paper describes an experiment intended to look for the first of these responses in two species of mammals, the marsupial South American bush opossum (Monodelphis domesticus) and the placental Djungarian hamster (Phodopus sungorus). In another paper, we will report on an attempt to demonstrate the second category of magnetic response in mammals (manuscript in preparation).

Compass responses in mammals have been reported for humans and the European woodmouse, Apodemus sylvaticus (Baker, 1980; Mather \& Baker, 1981). In addition, Baker, Mather, and Kennaugh (1983) and Kirschvink (1981) report deposits of magnetite in humans that could serve as the basis for a magnetic field receptor. The fact that humans were the subjects of much of this work has resulted in its receiving particularly widespread attention. Two attempts to replicate Baker's work on human orientation have not confirmed his results (Adler \& Pelke,

The senior author expresses his gratitude to the late William T. Keeton for the opportunity to perform this work at Cornell University. While at Cornell, he was supported in part by NSF Grant BNS 78-13016 to W. T. Keeton. Russell Charif participated in early stages of this work, Virginia Hayssen and Rachel Levin provided the test animals, and Bruce Moore provided stimulating discussions on magnetic orientation. R. C. Madden's present mailing address is: Department of Biology, Marymount College, Tarrytown, NY 10591. J. B. Phillips's mailing address is: Department of Biology, Yale University, Box 6666, New Haven, CT 06511 .
1985; Gould \& Able, 1981). We know of no effort to replicate Mather and Baker's work on woodmice. Thus, the question of whether mammals demonstrate magnetic compass orientation remains unsettled.

In the present experiment, we attempted to condition two species of mammals to search for food in the north direction of a magnetic field similar to the earth's. A system of coils made it possible to align the magnetic field so that north coincided with any one of the four arms of a cross-shaped maze; the subject's task was to pick the correct arm. The rationale behind the choice of the two species is presented in the discussion.

\section{METHOD}

A four-arm maze, $102 \mathrm{~cm}$ across, was constructed of acrylic plastic and glass (Figure 1). An automatic feeder that dispensed mealworms (for the opossum) or halves of sunflower seeds (for the hamster) was located at the end of each arm. The beam of an infrared photorelay was positioned across each arm so that it would be cut by an animal's approaching the delivery spout of the respective feeder. The central square of the maze presented the animal with the choice of either an overhead or underfloor nest box, both opening into the center of the maze. The crossed beams of two infrared photorelays detected the subject's entry into the central square from either nest box or any arm.

The maze was positioned at the center of two cube coils, which produce a more uniform magnetic field than the commonly used Helmholz coils (Rubens, 1945). The coils were wound on a common cubical nonmagnetic frame, $1.22 \mathrm{~m}$ on a side, so that they generated their magnetic fields in the horizontal plane, at right angles to each other, and, respectively, $135^{\circ}$ east and west of the north direction of the horizontal component of the earth's field. The current through each coil was set to produce a field strength about 1.41 times greater than that of the horizontal component of the earth's field (Phillips, 1985). 


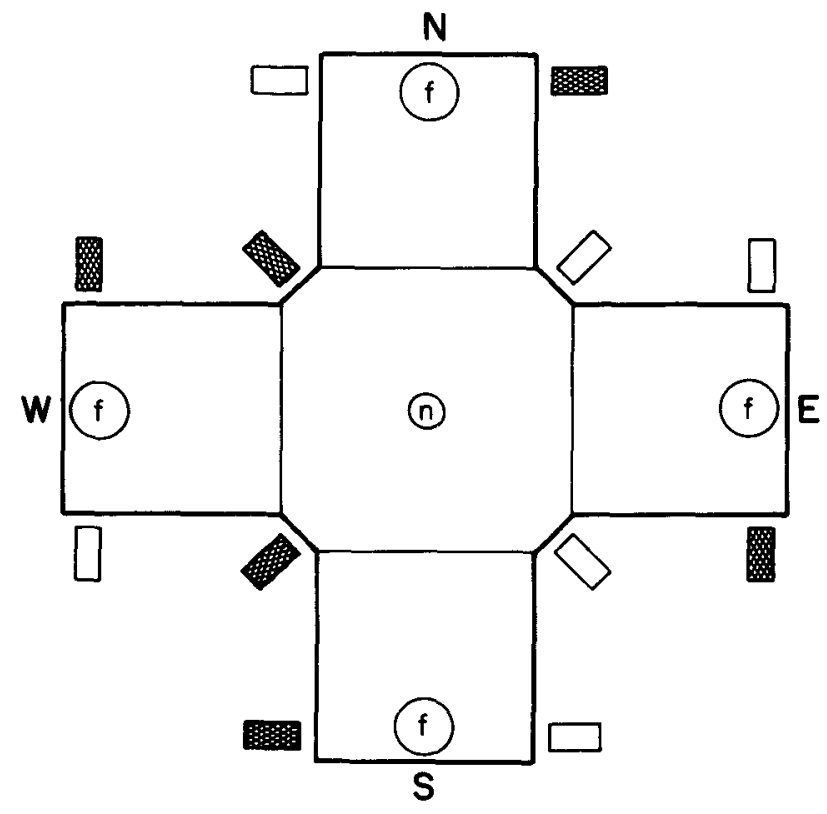

Figure 1. Floor plan of the maze. The lighter lines indicate a 7.5-cm step down from the central area into each arm. Small open rectangles are infrared beam sources. Small hatched rectangles are photorelay receivers. The letter $n$ designates nest chamber openings (one from top, one from bottom). The letter $f$ designates electromechanical food dispensers. $N, E, W$, and $S$ show the magnetic compass directions in the earth's natural field: cube coils allowed the north direction of the field to be pointed toward any arm (see text).

The maze was aligned with its four arms in the four cardinal magnetic compass directions, north, south, east, and west. With both coils off, the animal was exposed to the normal earth's field. Switching on one or the other coil produced an apparant field of intensity equal to the earth's, but rotated $90^{\circ}$ left or right. Switching on both coils produced a field of intensity equal to the earth's, but rotated $180^{\circ}$. In this manner, the north direction of an earth-intensity field could be pointed toward any arm.

A solid state logic-gate sequencer controlled the experiment and recorded the results on a multichannel strip-chart event recorder. Sequencer and recorder were located in a hallway adjacent to the room containing the maze, so that sounds generated by the recording pens and by the electromechanical relays that switched the currents to the coils were unlikely to be heard by the subject. (We had intended to do instrument controls to be certain of this point, but these were rendered unnecessary by the negative results.)

When the animal entered the central square of the maze and broke the beam of either of the crossed photorelays, the sequencer randomly selected one of the four possible field directions, switching the coils appropriately to produce the proper field. In one protocol, if the animal entered the arm in the north direction of the field and broke the corresponding photorelay beam, it received a food reward. Otherwise it did not. A given field was held until the animal responded in the proper arm, received its food reward, and returned to the central square, but it was scored as correct only if the first arm it entered was the one in the north direction of the field; otherwise it was scored as incorrect.

In most of the experiments with opossums, several consecutive trips (two to nine) between the central square and the end of an arm were required before it was considered that the animal had made a response. The animal was scored as correct if the first arm into which it made the proper number of consecutive entries was the arm in the north direction of the field; otherwise it was scored as incorrect. This protocol had the effect of increasing the effort involved in each response (and hence the penalty for responding on an incorrect arm), increasing the time of exposure to a given field (important because of suggestions that magnetic orientation may require integration of information over a long time period; Kreithen \& Keeton, 1974) and increasing the amount of movement through the magnetic field (movement is essential to some suggested mechanisms of magnetic field detection). It also prevented the animals from taking up a stereotyped circling motion around the maze-a strong behavioral tendency. The opossums readily learned the need for multiple entries. We usually initiated the animal to the apparatus with the single-entry protocol, and then increased the number of entries required. The multiple-entry protocol was not used with the hamsters because none of several individuals we tried could learn it.

A number of variations on these protocols were tried. For clarity, they are described for each series in the Results section.

The subjects were kept on a daily cycle of $12 \mathrm{~h}$ of bright light (two $100-\mathrm{W}$ bulbs about $2 \mathrm{~m}$ distant) and $12 \mathrm{~h}$ of dim light (one 7.5-W bulb centered $1.5 \mathrm{~m}$ over the maze). As both species are nocturnal, testing was done during the dim-light phase. A subject spent between 4 and 35 days in the maze. Any time the animal was in the maze but not actually being tested, the coils were set to produce a null horizontal field. This procedure was intended to prevent the animal's associating a particular arm of the maze with the north direction of the field. The first night was considered a period of familiarization and learning, and was not counted in the tabulation of results. On some nights, a malfunction in the apparatus (most frequently the blocking of one or more photorelay beams by stray nest material) prevented data collection.

The experiment was conducted in a relatively featureless room, unfurnished and without windows. When the animals were actually working, the room was dark except for the dim light over the maze. No elaborate precautions were taken to avoid biasing topographic features that the animals might perceive. However, examination of preliminary data indicated no directional bias in the animals' behavior. The location of the experiment was the Liddell Laboratory building of Cornell University, Ithaca, New York, $42^{\circ} 29^{\prime}$ North, $76^{\circ} 26^{\prime}$ West.

\section{RESULTS}

\section{Bush Opossum}

Seven series were run. The results are shown graphically on a night-by-night basis in Figure 2 (a-f) and Figure 3a, and a summary for each series is presented below. The summary is expressed as the number of correct responses over the total number of trials, followed by the result of chi-square testing against the expected 1:4 ratio for random responding.

Series 1 (Figure 2a). The subject was an adult female. Single entries into an arm were required. Duration of the series was 8 days, with data obtained on 6 nights. Result: $65 / 257(p>.05)$.

Series 2 (Figure 2b). The subject was the same as in the previous series. Multiple consecutive entries into an arm were required, starting with two, increasing to five, then decreasing to three near the end of the series. The duration of the series was 34 days, with data obtained on 26 nights. Result: 299/1127 ( $p>.05$ ).

Series 3 (Figure 2c). The subject was an adult male. Two consecutive trips into an arm were required. Duration was 4 days, with data obtained on 4 nights. Result: $29 / 117(p>.05)$. 
Series 4 (Figure 2d). The subject was a second adult male. The protocol was as in the preceding series, except that three arm entries were required on the final night. Duration was 4 days, with data obtained on 4 nights. Result: $72 / 261(p>.05)$.

Series 5 (Figure 2e). The geomagnetic field is horizontal $\left(0^{\circ}\right)$ at the magnetic equator, becomes increasingly inclined toward the magnetic poles, and is vertical at the poles. (The magnetic equator and poles are near their geographic counterparts, but not coincident with them.) Since the bush opossum is a tropical species, it would have encountered a less inclined field in its ancestral geographic range than it does in Ithaca, where the field inclination is about $72^{\circ}$.

An influential model developed with birds postulates that the direction of the magnetic field is sensed by means of a determination of the azimuth of the vertical plane onto which the magnetic field projects at the most acute angle to the horizontal (Wiltschko \& Wiltschko, 1972). In this case, it is possible, in a sendentary species, that selection could optimize the magnetic receptors to respond maximally at the field inclination of the species' ancestral geographic range.

Accordingly, for this series, we tested an opossum in a magnetic field whose inclination was reduced to $35^{\circ}$ by a vertical winding on the cube-coil frame that cancelled
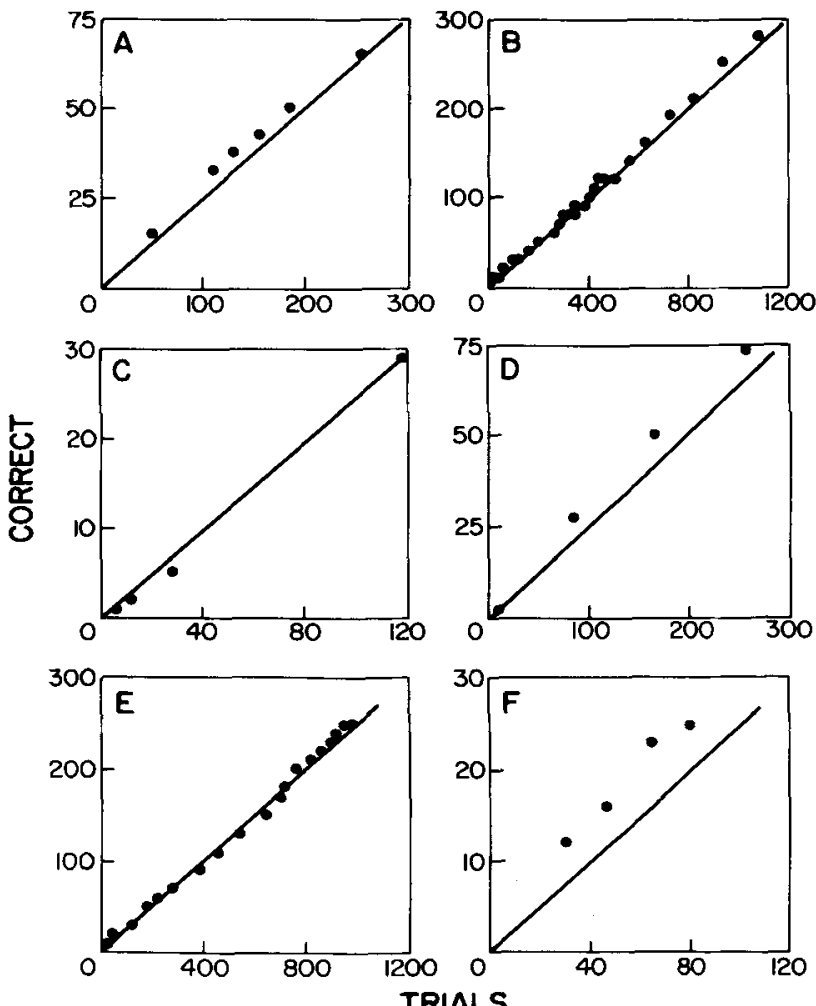

Figure 2. Plots of correct responses versus total responses. Each point indicates one night of data. Vertical and horizontal axes are scaled 1:4, so that the $45^{\circ}$ diagonal lines shown represent the random level of response.
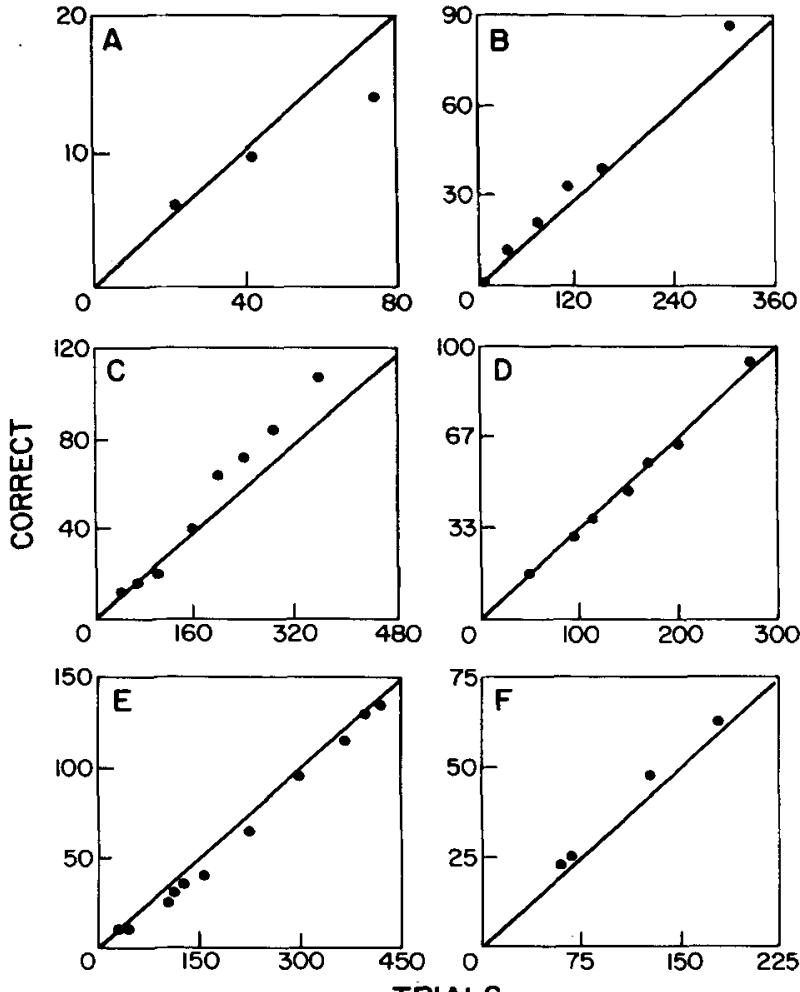

TRIALS

Figure 3. Plots of correct responses versus total responses. Representation is as in Figure 2, except that the random level of response is $1: 3$ for $D-F$ (see text).

part of the vertical component of the earth's field in Ithaca. The subject was a new adult female. The series duration was 31 days, with data collected on 20 nights. Initially, two consecutive entries into an arm were required; this was gradually increased to nine during the series. Result: $253 / 972(p>.05)$.

Series 6 (Figure 2f). In this series, a high-pitched (about $3000 \mathrm{~Hz}$ ) "buzzer" sounded for 5 to 10 sec on an incorrect response to increase feedback to the animal. While the buzzer was sounding, the apparatus was deactivated so that the subject could not trigger a photorelay in another arm of the maze. The vertical coil was not used. The subject was the same as in the previous series. First one, then two entries into an arm were required. The series duration was 4 days, with data obtained on 4 nights. Result: $25 / 79(p>.05)$.

Series 7 (Figure 3a). The protocol was the same as in Series 6 , but two arm entries were required throughout. The subject was a new adult female. The series duration was 3 days with data collected on 3 nights. Result: 14/73 $(p>.05)$.

\section{Djungarian Hamster}

Five series were run. Multiple arm entries could not be used as explained previously, but the sounding of a $3000 \mathrm{~Hz}$ buzzer for $5 \mathrm{sec}$ on an incorrect response was used as indicated below. The presentation of the results is as for the opossum (Figures 3b-3f). 
Series 1 (Figure 3b). The subject was a young male. The series duration was 10 days, with data obtained on 6 nights. The buzzer was used on Nights 4 through 6 . Result: 88/306 ( $p>.05$ ).

Series 2 (Figure $3 c$ ). The subject was a second young male. The buzzer was used throughout. The series duration was 11 days, and data were obtained on 8 nights. Result: $106 / 354$ (.05 > $>$ >025).

Series 3 (Figure 3d). In the previous series, we observed that hamsters almost never made sequential responses in the same arm. Thus, the subject almost invariably made a wrong response if the sequencer repeated a field. To take this behavioral trait into account, we set the sequencer so that it would not select the same field consecutively. Since, for each trial, the possible correct arms were the three other than the one previously selected, the random level of response was 1:3 instead of 1:4. In the rare cases $(<1 \%)$ in which the animal did make its first response on the previously selected arm, the response was discounted. That is, the animal was scored on the basis of its first response in an arm that was not a repeat of the correct arm for the previous trial. The subject for this series was the same as in Series 2. Series duration was 9 days, with data obtained on 6 nights. The buzzer was used throughout. Result: $92 / 275(p>.05)$.

Series 4 (Figure $3 \mathrm{e}$ ). The protocol was the same as in Series 3, but without the buzzer. The subject was a new male. Series duration was 15 days, with data collected on 11 nights. Result: $134 / 418(p>.05)$.

Series 5 (Figure $3 f$ ). The protocol was the same as in Series 3 , including the buzzer. The subject was the same as in Series 4. Series duration was 4 days, with data collected on 4 nights. Result: 62/181 ( $p>.05)$.

\section{DISCUSSION}

Opossums performed at a random level in all series. Hamsters performed at a random level in four out of five series. A significant result was obtained in Series 2, but examination of Figure 3c shows this to be attributable to an above average performance on a single night (Night 5). There is no general pattern of above average or improving performance, and so this single night is clearly an instance of a random deviation, likely to occur in any substantial body of data.

The decision to use a marsupial for part of this experiment stemmed from the consideration that marsupials have a retina that includes double cones, a trait shared with most other vertebrates, including birds. This contrasts with the single-cone-only retina of placental mammals. An important model for a magnetic detection mechanism is the optical pumping theory of Leask (1977). Since the largest body of evidence for magnetic orientation comes from birds, and since, if the Leask model should prove correct, retinal structure and function could be important in determining whether an animal can sense the magnetic field, it appeared appropriate to test a mammal with a retina more similar to that of birds.

The Djungarian hamster was chosen for its small size (considerably less than that of the more familiar Siberian hamster). Our maze was relatively larger in comparison to this species than to any other species of placental mammal readily available to us. This maximized the animal's effort in searching for food and the relative amount of movement through the magnetic field, possible factors in determining whether an animal is motivated to use magnetic orientation, or able to do so.

Overall, our results do not confirm the hypothesis of a magnetic compass sense in mammals. It seems appropriate to place this finding in the context of other attempts to demonstrate geomagnetic sensitivity in animals that were not free-ranging.

Most reports of success come from situations in which three conditions existed: (1) a compass response was involved, (2) the subject was able to move while making the discrimination, and (3) the direction of the response was acquired outside the experimental situation, that is genetically programmed, imprinted, or learned over possibly a lengthy time period in the natural environment. This is the case for the various experiments based on migratory or homing orientation (e.g., Emlen, Wiltschko, Demong, Wiltschko, \& Bergman, 1976; Mather \& Baker, 1981; Wiltschko \& Wiltschko, 1972). In the case of the work by Baker (1980) on humans, the subjects were not moving at the moment the response was made but were transported in the earth's magnetic field just prior to making the response; otherwise, the above conditions were met.

Three studies on birds in which the subjects were immobilized and were to make a nonorientational conditioned cardiac response to the magnetic field did not demonstrate magnetic sensitivity (Beaugrand, 1976, 1977; Kreithen \& Keeton, 1974). Reille (1968) had reported positive results with this experiment, but the negative results of the three subsequent efforts make this finding doubtful.

In the present experiment, the animals were making a compass response, and were able to move, but learned the direction of the response as part of the experiment. Possibly this feature is critical. On the other hand, our subjects, who were always required to respond in the apparent north direction of the magnetic field, were given rather substantial periods of time to learn the response in some series. Also, not every reported successful demonstration of magnetic sensitivity in confined animals meets the conditions stated above. Bookman (1978) reports a successful experiment on pigeons in which they learned to respond to the presence or absence (but not the orientation) of a magnetic field. The pigeons were able to fly while making the discrimination. Phillips (1977, 1985) has obtained evidence of a trained preference for movement in a direction relative to that of an earthstrength magnetic field in two species of salamanders.

In short, the conditions in our experiment do not violate in any clear way conditions that have been previously reported to result in successful magnetic orientation by confined subjects. In addition, learning to search for food in a particular direction of the magnetic field does not 
seem to us to be an adaptively implausible use for magnetic sensitivity. On the contrary, being able to orient magnetically when celestial light cues are obscured during foraging would appear to be a useful capability, especially in a nocturnal species.

There are, of course, various other reasons why our experiment might have failed to reveal an existing magnetic sensitivity. In particular, although the animals could move, they could not range over distances that would be normal in the wild. It is conceivable that more normal movements might be required either as part of the detection mechanism or as a necessary condition for paying attention to the magnetic field. Obviously, moving around a small, four-arm maze poses nothing like the orientational problem of moving about a normal home range.

In addition, due to distortion of the geomagnetic field by ferrous materials in the building and generation of electromagnetic fields by electrical wiring, lights, equipment, and so forth, magnetic and electromagnetic field conditions in the building housing the apparatus were not entirely natural. Despite these various considerations, our results reduce confidence in the hypothesis of mammalian ability to orient to earth-strength magnetic fields.

\section{REFERENCES}

ADler, K., \& Pelke, C. (1985). Human homing orientation: Critique and alternative hypotheses. In D. S. Jones, B. J. MacFadden, \& J. L. Kirschvinck (Eds.), Biomagnetism. New York: Plenum.

BAKER, R. R. (1980). Goal orientation by blindfolded humans after longdistance displacement: Possible involvement of a magnetic sense. Science, 210, 555-557.

Baker, R. R., Mather, J. G., \& Kennaugh, J. H. (1983). Magnetic bones in human sinuses. Nature, 301, 78-80.

Beaugrand, J. P. (1976). An attempt to confirm magnetic sensitivity in the pigeon, Columba livia. Journal of Comparative Physiology, A 110, 343-355.

Beaugrand, J. P. (1977). Test of magnetic sensitivity in seven species of European birds using a cardiac nociceptive conditioning procedure. Behavioral Processes, 2, 113-127.

Bookman, M. A. (1978). Sensitivity of the homing pigeon to an earth strength magnetic field. In K. Schmidt-Koenig \& W. T. Keeton (Eds.), Animal migration, navigation, and homing (pp. 127-134). Berlin: Springer-Verlag.

Emlen, S. T., Wiltschko, W., Demong, N. J., Wiltschko, R., \& Bergman, S. (1976). Magnetic direction finding: Evidence for its use in migrating indigo buntings. Science, 193, 505-507.

Gould, J. L., \& ABLE, K. P. (1981). Human homing: An elusive phenomenon. Science, 212, 1061-1063.

Kirschvink, J. L. (1981). Ferromagnetic crystals of magnetite in man. Journal of Experimental Biology, 92, 333-335.

Kreithen, M. L., \& Keeton, W. T. (1974). Attempts to condition homing pigeons to magnetic stimuli. Journal of Comparative Physiology, A 91, 355-362.

LEASK, M. J. M. (1977). A physicochemical mechanism for magnetic field detection by migratory birds and homing pigeons. Nature, $\mathbf{2 6 7}$ 144-145.

MAther, J. G., \& BaKer, R. R. (1981). Magnetic sense of direction in woodmice for route-based navigation. Nature, 291, 152-155.

PHILlips, J. B. (1977). Use of the earth's magnetic field by orienting cave salamanders (Eurycea lucifuga). Journal of Comparative Physiology, A 121, 273-288.

Phillips, J. B. (1985). Magnetic compass orientation in the eastern redspotted newt (Notopthalmus viridescens). Joumal of Comparative Physiology, A 158, 103-109.

REILLE, A. (1968). Essai de mise en évidence d'une sensibilité du pigeon au champ magnétique à l'aide d'un conditionnement nociceptif. Joumal of Physiology (Paris), 60, 85-92.

RUBENS, S. M. (1945). Cube surface coil for producing uniform magnetic fields. Review of Scientific Instruments, 16, 243-245.

Wiltschko, W., \& Wiltschko, R. (1972). Magnetic compass of European robins. Science, 176, 62-64.

(Manuscript received November 12, 1985; revision accepted for publication November 15, 1986.) 